\title{
Aprendizagem com Robótica Educacional: uma Abordagem Baseada em Problemas
}

\author{
Cristiane Pelisolli Cabral ${ }^{1}$, Evandro Preuss ${ }^{1}$, Liliana Passerino ${ }^{1}$ (In Memoriam) \\ ${ }^{1}$ Universidade Federal do Rio Grande do Sul - UFRGS \\ Porto Alegre - RS - Brasil \\ pelisolli@gmail.com, evandro.preuss@gmail.com
}

\begin{abstract}
Educational robotics can be used efficiently in the teaching and learning processes for the improvement of computational thinking and also for the formation of scientific concepts of non-robotic areas. Problem-based learning can use robotics to think and develop solutions to these real-world problems, making it necessary to know the problems and appropriate the concepts involved in order to develop its solution, and in this process, robotics enters as a factor motivating and attractive. This work presents a case study with the programming of robots with a solution based on free hardware, where programming challenges were launched to solve the problems involving ecology.
\end{abstract}

Resumo. A robótica educacional pode ser utilizada de modo eficiente nos processos de ensino e aprendizagem para o aprimoramento do pensamento computacional e também para a formação de conceitos científicos de áreas que não são da robótica. A aprendizagem baseada em problemas pode utilizar a robótica para pensar e desenvolver soluções para esses problemas do mundo real, fazendo com que seja necessário conhecer os problemas e se apropriar dos conceitos envolvidos para poder desenvolver sua solução e nesse processo, a robótica entra como um fator motivador e atrativo. Este trabalho apresenta um estudo de caso com a programação de robôs com uma solução baseada em hardware livre, onde foram lançados desafios de programação para a resolução dos problemas envolvendo a temática da ecologia.

\section{Introdução}

O uso de recursos tecnológicos, como a robótica educacional, vem sendo explorado há algum tempo para o suporte ao ensino e à aprendizagem e está se consolidando cada vez mais como um recurso capaz de motivar e fomentar processos de aprendizagem para o desenvolvimento de habilidades cognitivas e sociais, bem como do pensamento computacional (Avila, 2017; Oliveira, 2017).

As formas de aplicação de recursos tecnológicos nas atividades educacionais são baseadas em diferentes fundamentações teóricas e com diversas técnicas e metodologias. O construtivismo e o construcionismo ou uma combinação desses conceitos teóricos e abordagens predominam a pesquisa e experimentos sobre pensamento computacional utilizando robótica educacional (Ioannou, A. \& Makridou, 2018). 
Um dos principais problemas encontrados no uso de informática na educação, especialmente de robótica educacional, é que uma grande parte das propostas se baseia no ensino e uso da própria tecnologia e sua aplicação fica restrita às áreas de ciências, tecnologia, engenharia e matemática (Liying Xia, Baichang Zhong, 2018), mesmo que de forma interdisciplinar (Eguchi, 2014).

Inúmeras escolas possuem disciplinas e oficinas que ensinam robótica educacional, inclusive com participação em competições de robótica, porém a grande maioria delas utilizam kits de robótica comerciais e de alto custo para desenvolver soluções típicas de competição, como andar em um cenário, competição de sumô, futebol e dança. No entanto, poucas utilizam a robótica para ensino de outros conteúdos e conceitos científicos, da mesma forma que o uso de hardware livre para robótica ainda é pouco explorado.

Este trabalho apresenta um estudo de caso realizado com quatro alunos dos anos finais do Ensino Fundamental, já familiarizados com a construção e a programação de robôs, com uma solução baseada em hardware livre. Foi proporcionado aos estudantes uma experiência com uma ferramenta de robótica educacional onde foram lançados desafios de programação em duplas para a resolução de problemas envolvendo o ambiente de um parque ecológico. Posteriormente, foi analisado o processo de aprendizagem desses estudantes com base na Epistemologia Genética de Jean Piaget, no construcionismo de Papert e na abordagem baseada em problemas.

\section{Aprendizagem ativa}

Para Piaget (1975), a inteligência possui duas funções chamadas de invariantes funcionais, por manter seu funcionamento invariável desde as fases mais primitivas do desenvolvimento, que são a adaptação e a organização. Adaptação e organização são inseparáveis, pois o primeiro refere-se ao funcionamento de um sistema e o segundo às suas estruturas. Segundo Piaget (1975), a adaptação é um equilíbrio entre assimilação e acomodação, porém, quando nos deparamos com algo novo para nossa inteligência, algo que desconhecemos, tentamos assimilar esse objeto com os esquemas e estruturas cognitivas que possuímos, mas nem sempre é possível, por isso realizamos modificações estruturais para que o conhecimento novo possa ser incorporado. Quando não há possibilidade de assimilação, o esquema se acomoda adaptando-se, para se transformar num outro mais adequado e capaz, então, de realizar a assimilação. Ao acomodar, o esquema se reconstrói e se prolonga gerando um novo esquema de assimilação em outro nível, sempre mais elevado, de equilíbrio. Portanto, a atividade assimiladora do sujeito se complementa na acomodação, quando acontece a transformação das estruturas cognitivas e consequentemente, a aprendizagem.

Nesse sentido, pensamos no aluno/estudante como o agente responsável por sua aprendizagem através da busca constante da construção da sua aprendizagem, como nos diz Moran (2015) e as metodologias ativas. Jean Piaget, teórico de referência da Epistemologia Genética, dedicou suas pesquisas para explicar as origens e os mecanismos da construção do conhecimento. Piaget escreveu ao longo de suas obras que a origem do conhecimento é uma construção realizada através das ações físicas (agindo sobre os objetos) e mentais (reorganizando as estruturas mentais) do sujeito. Para aprender, cada sujeito construirá para si os objetos do meio e isso acontecerá 
através da sua própria ação física e mental, por isso a aprendizagem é um processo ativo. Para realizar todo o processo de construção do conhecimento é necessário que o sujeito aja externamente sobre objetos e opere internamente nas suas estruturas mentais. Ainda que as metodologias ativas $(\mathrm{BACICH}, 2018)$ possam parecer algo bastante inovador, elas possuem suas bases nas diretrizes propostas por teóricos interacionistas bastante estudados como Piaget, Papert, Vigostsky e Paulo Freire. Atualmente, tentamos aplicar essas teorias para explicar mecanismos cognitivos em interação com esses objetos que não existiam naquele tempo. Por isso, a relevância da discussão acerca das metodologias ativas.

Seymour Papert, na década de 1960-70, vislumbrou o potencial educativo do computador para a aprendizagem escolar ativa. O pesquisador dedicou-se a criar uma linguagem de programação na qual crianças "ensinavam" os computadores. O pesquisador acreditava que a educação seria potencializada com a possibilidade de alunos "ensinando" (programando) máquinas (computadores). Com referência na Epistemologia Genética de Piaget, Papert cunhou o termo "Construcionismo" (Papert, 2008) em oposição ao "Instrucionismo" para identificar a maneira como professores deveriam atuar em sala de aula, que no seu ponto de vista, deveria ser com menos fala e mais ação dos alunos. Ele criou a linguagem de programação chamada LOGO (Papert, 1985) ou "linguagem da tartaruga" como ficou popularmente conhecida e estabeleceu relações entre a ferramenta LOGO e a aprendizagem humana através da Epistemologia Genética de Jean Piaget. A ferramenta proporciona que crianças possam programar os movimentos de uma tartaruga em uma tela de computador utilizando comandos simples e dessa forma, através da ação física e mental, construir hipóteses, testar e reconstruí-las imediatamente. Na época em que o computador era pouco utilizado para fins educacionais, Papert projetou um futuro próximo onde as crianças cresceriam manuseando computadores. Ele acreditava que os meios educacionais possuíam poucos objetos concretos para ajudar os alunos a construírem seus conhecimentos. Por isto, dedicou-se a investigar sobre recursos que ajudassem alunos a pensar ativamente ou "objetos para pensar com", ou seja, objetos concretos que estimulassem a criança a pensar sobre o pensar e dessa forma testar hipóteses através da exteriorização das mesmas.

\section{Robótica educacional e aprendizagem}

Com o passar do tempo, observando o uso que se fez da ferramenta LOGO em sala de aula, a experiência mostrou que a tela do computador não era algo tão concreto para a criança como se dimensionou. Por isso, à linguagem LOGO ou "linguagem da tartaruga" Papert uniu peças do tipo LEGO®, que proporcionaram a construção de objetos concretos que realizam movimentos no mundo real tal como a tartaruga realizava no mundo virtual. Iniciou-se então o Projeto LEGO-LOGO no Laboratório de Mídias do Massachusetts Institute of Technology (MIT / EUA), com ajuda de seus colaboradores e convênio com a LEGO ${ }^{\circledR}$ Dacta, que concretizou a "caixa de engrenagens" idealizada por Papert no kit de robótica educacional LEGO® Mindstorms. Através deste kit, a tartaruga programável, idealizada por Papert, agora está fora da tela do computador e no formato criado pela criança. Ela pode ser construída com os blocos plásticos na forma de um carro, uma moto, um animal, ou qualquer outro protótipo imaginado pela criança, e em seguida programada pela linguagem inspirada no LOGO. 
Na década de 1970-80 estão as origens do trabalho com robótica educacional no ambiente escolar e desde então procura-se investigar o potencial educativo da ferramenta, para aperfeiçoar processos de ensino e aprendizagem utilizando esse recurso tecnológico. Alguns trabalhos acadêmicos realizaram uma leitura da aprendizagem com base na Epistemologia Genética de Piaget relacionando com a atividade de robótica educacional no meio escolar. É o caso do trabalho de Oliveira (2007) que teoriza acerca dos processos cognitivos com ênfase na Tomada de Consciência (Piaget, 1977) de estudantes utilizando a ferramenta da robótica. Lopes (2008) desenvolveu investigação acerca da aprendizagem enfatizando o processo de Abstração Reflexionante (Piaget, 1995). Além dessas investigações, Stefen (2002), Zilli (2004) e Ribeiro (2006) também contribuem para a teorização da robótica no âmbito escolar, como no caso do último estudo que relata o desenvolvimento de um projeto de aprendizagem com robótica educacional realizado no ensino fundamental.

Muitas discussões recentes acerca do ensino de robótica e programação envolvem o conceito de pensamento computacional, que seria uma habilidade a ser desenvolvidas nos estudantes através do ensino utilizando recursos tecnológicos. A necessidade do mercado de trabalho, cada vez maior, de mão de obra para profissões relacionadas à área da Ciência da Computação faz com que se reflita acerca dos processos de ensino e aprendizagem no âmbito escolar para que não se forme uma população de analfabetos digitais.

O pensamento computacional é a forma de pensar e resolver os problemas de modo que os mesmos possam ser representados e implementada por um humano ou computador (Wing 2006). A principal característica do pensamento computacional é a capacidade de pensar como um cientista da computação ao confrontar um problema (Barr e Stephenson, 2011), organizando o desafio e sua solução numa sequência de passos bem definidos e organizados que levam à solução. O Pensamento Computacional é um processo cognitivo bastante amplo que envolve habilidades como abstração, decomposição, identificação de padrões e construção do algoritmo de programação e sua definição conceitual ainda se encontra em processo de construção (Bocconi, 2016).

A Aprendizagem Baseada em Problemas (ABPA) ou Problem Based Learning $(\mathrm{PBL})$ é uma prática pedagógica empregada no ensino em diversas áreas e é perfeitamente adequado para ser usado com robótica educacional e para desenvolver o pensamento computacional. Na matemática destaca-se a abordagem de Pólya para resolução de problemas. Segundo Pozo (1998), George Pólya (1887-1985) foi um matemático húngaro que trabalhou numa grande variedade de tópicos matemáticos, que incluíam séries, teoria dos números, combinatória, e teoria das probabilidades. Entre suas produções, tentou caracterizar o modo como se resolvia problemas de matemática e tentou descrever como deveria ser ensinada a resolução de problemas.

Os passos sugeridos pelo autor para a resolução de problemas em matemática são os seguintes: 1) Compreender o problema; 2) Conceber um plano encontrando conexão entre os dados e os desconhecidos. Considerar problemas auxiliares se uma ligação imediata não pode ser encontrada; 3) Realizar o plano; 4) Avaliar, examinando a solução obtida. Os passos propostos para a ABPA ou PBL, ainda que não sejam as mesmas etapas propostas por Wing (2006) para o Pensamento Computacional, parecem ter algumas relações. 
VIII Congresso Brasileiro de Informática na Educação (CBIE 2019)

Anais dos Workshops do VIII Congresso Brasileiro de Informática na Educação (WCBIE 2019)

\section{Contexto da pesquisa}

A ideia inicial para a aplicação desse experimento teve como motivação inicial o projeto PITAIA - Proposta Inovadora de Tecnologia Assistiva para Inclusão e Aprendizagem em ciências para alunos com deficiência na comunicação. Os autores do presente artigo foram alunos de uma disciplina onde conheceram o projeto, que tem como objetivo desenvolver um ambiente de ensino tridimensional tangível voltado ao ensino de ciências com uso integrado de comunicação alternativa em interfaces interativas tangíveis, robótica e de realidade virtual, que propicie processos de construção de conceitos científicos com crianças com deficiência na comunicação, matriculadas nos anos finais do ensino fundamental da educação básica.

O estudo de caso (YIN, 2016) envolveu a contextualização do problema sobre preservação ecológica de um parque estadual, a sistematização da solução para resolver alguns problemas através da programação e o uso de um robô educacional. Participaram quatro alunos de anos finais do ensino fundamental, entre 13-14 anos de idade, da rede pública de uma cidade capital de estado, que já tinham algum conhecimento de robótica educacional. Esses alunos foram divididos em dois grupos.

\subsection{Problematização}

Um mapa do parque ecológico impresso em papel e colocado sobre a mesa foi o cenário para a formação de conceitos científicos e o pensamento computacional, onde foram apresentados os principais problemas do parque ecológico, que é a preservação do mesmo e a manutenção das espécies nativas. Este parque enfrenta problemas devido ao lixo deixado pelos visitantes e uma de suas espécies - um sapinho - está em extinção.

Inicialmente foram apresentados o robô educacional e o ambiente de programação, onde os alunos puderam testar os comandos e movimentos básicos de um robô através do ambiente de programação. Na sequência, foi apresentado o parque ecológico, tema do estudo, com a descrição do mesmo e os seus principais problemas, envolvendo a preservação ambiental e a manutenção de suas espécies nativas.

A partir dessa contextualização foram lançados desafios em que era necessário utilizar a robótica para pensar em soluções que pudessem resolver ou amenizar os problemas identificados. Uma lista de possíveis soluções e implementações robóticas foi elaborada e dentre elas, de acordo com as limitações do material educacional disponível, duas delas foram escolhidas para serem implementadas: um robô coletor de lixo e um robô para encontrar o sapinho. $\mathrm{O}$ ambiente de programação foi utilizado para desenvolver os programas e transferir os códigos para os robôs, que foram executados no cenário que simulava o ambiente do parque.

\subsection{O material}

A ferramenta utilizada nesta investigação foi o robô MBot (figura 1), que foi criado pela empresa chinesa mBlock. O robô é apresentado no site como sendo utilizado por mais de 4,5 milhões de crianças pelo mundo. O mBot (Robot Kits for Kids) é um robô educacional para iniciantes, que torna o ensino e aprendizado de programação de robôs mais lúdico e simples, através da linguagem de blocos. Com as instruções passo a passo e um cronograma de estudo fornecidas pelo produto, as crianças podem construir um robô e testar suas hipóteses através do material concreto. Assim, eles podem aprender 
VIII Congresso Brasileiro de Informática na Educação (CBIE 2019)

Anais dos Workshops do VIII Congresso Brasileiro de Informática na Educação (WCBIE 2019)

sobre uma variedade de maquinário robótico, peças eletrônicas e fundamentos da programação baseada em blocos, possibilitando o desenvolvimento do raciocínio lógico e habilidades de design.

O material, baseado em Arduino, é composto por uma base de metal, duas rodas, sensor de luz, sensor ultrassônico e seguidor de linha, mas permite expansão. O produto é recomendado para crianças a partir dos 8 anos de idade. A plataforma pode ser integrada a outra ferramenta de programação como a LEGO ${ }^{\circledR}$ Mindstorms, sendo possível integrar peças, sensores e lâmpadas. É vendido no site da Amazon por U\$79.

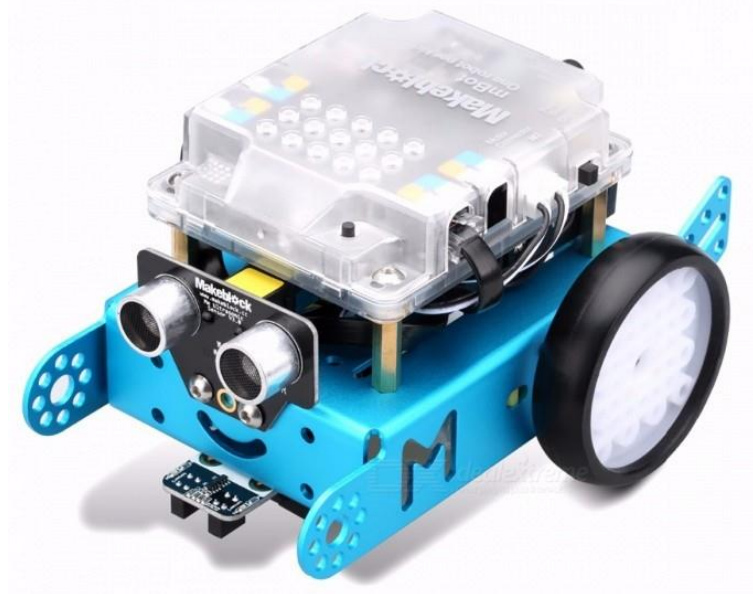

Figura 1. Robô MBot,

O ambiente de software de programação é o mBlock (mBlock, 2019), que é uma interface gráfica baseada no Scratch Open Source Code, que facilita a programação de projetos Arduino e interativos. A criança pode escrever o código através de blocos, mas também pode visualizar a programação baseada em Arduino ao lado.

\subsection{Atividades Desenvolvidas}

A atividade foi desenvolvida durante dois dias seguidos de trabalho das $14 \mathrm{~h}$ às $18 \mathrm{~h}$ (quatro horas de duração). No primeiro dia foi proporcionada a aproximação dos alunos com o material e foi apresentada a interface de programação e como construir o código.

Em seguida foi lançado o desafio para que os alunos dominassem os movimentos possíveis do robô educacional (fig. 2). Os problemas e desafios do primeiro dia foram:

- movimentar o robô para frente e para trás;

- movimentar o robô realizando um quadrado;

- movimentar o robô para frente e parar ao detectar a presença de uma mão na frente do sensor de presença; e

- movimentar o robô deslocando-se em cima de uma linha preta.

No segundo dia foi apresentado o contexto do parque ecológico e seus problemas ambientais onde foram lançados problemas/desafios para serem solucionados através das soluções robóticas. Os problemas e desafios do segundo dia foram: 
VIII Congresso Brasileiro de Informática na Educação (CBIE 2019)

Anais dos Workshops do VIII Congresso Brasileiro de Informática na Educação (WCBIE 2019)

- movimentar o robô seguindo a linha preta e recolhendo o "lixo do parque" representado pelos clips metálicos;

- movimentar o robô no espaço retangular delimitado com linha preta recolhendo o "lixo do parque" representado pelos clips metálico; e

- movimentar o robô no espaço delimitado com linha preta e localizar o sapinho que está em extinção.

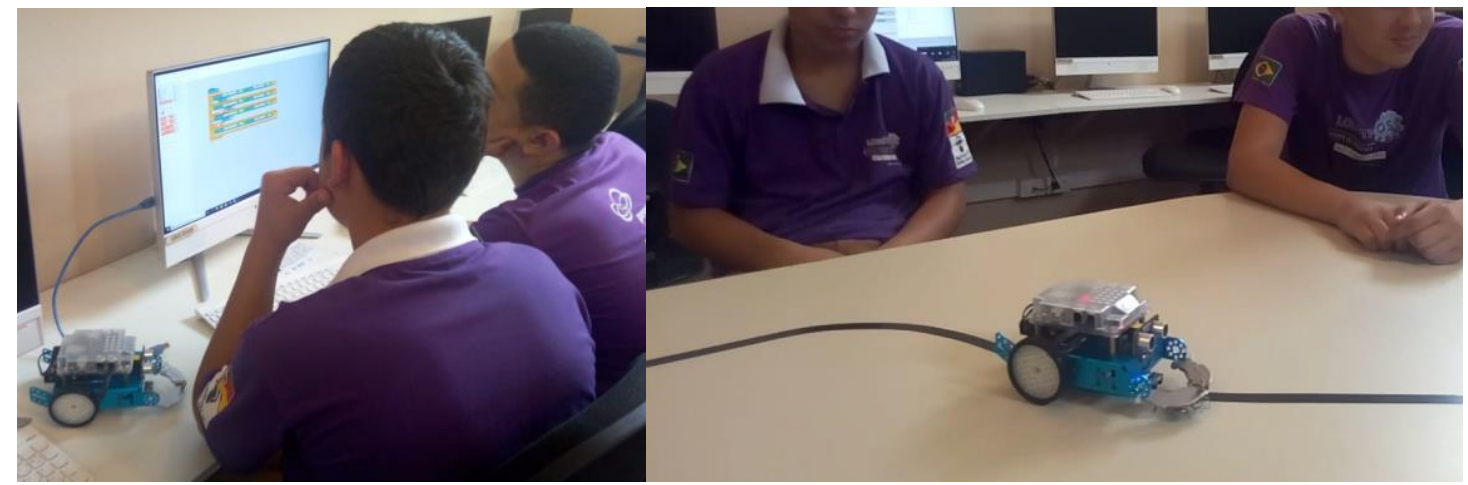

Figura 2. Uso do robô educacional

\section{Resultados e Discussões}

Os alunos conseguiram utilizar rapidamente o ambiente de programação baseado numa interface gráfica, com blocos de comandos para o robô, que são unidos formando uma sequência de instruções. Por já conhecerem programação LEGO ${ }^{\circledR}$ Mindstorms, também baseada em blocos, os estudantes adaptaram seus esquemas assimilatórios rapidamente para a interface de blocos no Scratch e aos comandos disponíveis na plataforma Arduino. Os alunos tentaram assimilar o conhecimento novo as estruturas construídas anteriormente, mas necessitaram realizar modificações estruturais para que 0 conhecimento novo pudesse ser incorporado. Pois como dito anteriormente, segundo Piaget (1975), a adaptação é um equilíbrio entre assimilação e acomodação, porém, quando nos deparamos com algo novo para nossa inteligência, algo que desconhecemos, tentamos assimilar esse objeto com os esquemas e estruturas cognitivas que possuímos, mas nem sempre é possível, por isso integramos um conhecimento novo aos esquemas que possuímos, reorganizando essas estruturas de maneira ascendente.

A estratégia utilizada pelos grupos para a resolução do problema aproximou-se muito da "tentativa e erro" como forma de experimentação. Observou-se que os alunos compreenderam o problema, elaboraram a solução baseada no código de programação e a partir daí foram realizando pequenos ajustes e testes até chegar na solução satisfatória para o problema. Observou-se também na conduta dos estudantes que os ajustes eram realizados um por um, não sendo possível para os sujeitos, naquele momento, fazer vários ajustes na programação para depois testar o robô em uma única vez. Tal constatação pode ter relação com o estágio do desenvolvimento cognitivo em que os sujeitos se encontram, visto que a aprendizagem é construída iniciando na atividade do "fazer concretamente" até chegar nas instâncias superiores do pensamento através da compreensão e representação por meio da linguagem. Esse processo é marcado pelo mecanismo de tomada de consciência como nos diz Piaget (1977). O auxílio de um objeto concreto para resolução de um problema de robótica educacional, pode ser 
relacionado também com "objetos para pensar com" da teoria construcionista de Papert (2008). São esses objetos que auxiliam o sujeito a refletir sobre o problema, reformular sua hipótese e testá-la novamente.

As duas duplas de alunos conseguiram movimentar o robô com algumas "tentativas e erros" de escrita do código. A utilização de blocos de comandos facilitou a programação pois não necessitou de cuidados com a sintaxe da linguagem, podendo focar a solução na resolução do problema e não nos detalhes da linguagem. Os erros frequentes na sintaxe da linguagem de programação podem levar o aprendiz a desistir de solucionar um problema, visto que o erro pode ser um elemento para aprimorar uma solução, mas em excesso pode levar à desistência do sujeito.

A contextualização da atividade através da temática do parque ecológico fez com que o experimento apresentasse mais sentido para os estudantes e mantivesse o interesse para a resolução dos problemas. A necessidade de identificar o problema e organizar soluções através da ferramenta da robótica educacional e da programação numa sequência ordenada de passos fez com que os estudantes fossem estimulados a desenvolver o pensamento computacional. Tal fato evidencia que a aprendizagem baseada em problemas com robótica educacional é uma abordagem que pode ser usada para desenvolver o pensamento computacional e pode ser usada para formar conceitos científicos envolvendo outros assuntos, para além da área de robótica.

Os alunos demonstraram grande interesse e engajamento durante todo o período de execução das atividades, o que demonstra que o uso de robótica nos processos de ensino aprendizagem é uma ferramenta interativa e atrativa que provoca a motivação do sujeito através da solicitação de sua ação física e mental para solucionar problemas. Dessa maneira, o estudante não é apenas um sujeito passivo no processo de ensino e aprendizagem, mas alguém que busca soluções para as suas hipóteses através de experimentações. Os alunos demonstraram iniciativa e pró-atividade durante todo o tempo, buscando criar e resolver as situações quase sem a necessidade de interferência dos instrutores, desenvolvendo uma solução, testando e refazendo até obter o resultado esperado.

Neste experimento foi utilizado um kit comercial Mbot baseado em Arduino por se tratar de uma plataforma disponível no laboratório, mas é perfeitamente possível e talvez mais interessante desenvolver e construir os robôs a partir de componentes da plataforma Arduino, com os alunos fazendo parte desse processo de montagem dos robôs, com a escolha dos componentes através da metodologia de resolução de problemas. Além disso, é importante ressaltar que o hardware livre possui um custo consideravelmente menor que as soluções comerciais convencionais.

\section{Conclusão}

O uso de robótica educacional com aprendizagem baseada em problemas do mundo real é uma abordagem ativa, interessante e viável para desenvolver o pensamento computacional e proporcionar a formação de conceitos científicos, pois ela é uma forma atrativa, interativa e motivadora para os alunos. Fazer o aluno agir, pensar e resolver problemas do mundo real, sistematizando uma sequência lógica de passos, que pode ser realizada por um robô, proporciona ao aluno conhecer com mais profundidade o problema e pode possibilitar a conceituação de conteúdos científicos. 
O uso de programação baseada em hardware livre, como o Arduino, é uma alternativa interessante, de baixo custo e que proporciona para o aluno todas as condições necessárias para aprender e desenvolver seus projetos de robótica, tanto os simples quanto os mais complexos, de modo interativo e intuitivo, com interfaces gráficas que não necessitam de habilidade em uma determinada linguagem de programação.

Como encaminhamento para trabalhos futuros, seria interessante desenvolver o próprio robô com os componentes de hardware livre, como os da plataforma Arduino, com a escolha dos componentes do robô como parte da resolução do problema. Outra alternativa seria desenvolver atividades introduzindo a programação com linguagem de programação ao invés dos blocos do Scratch, o que daria mais liberdade e possibilidades de programação, embora isso aumentasse o nível de dificuldade. Além disso, a atividade poderia ser utilizada para a sistematização dos passos para diferentes áreas, como geometria e física, por exemplo.

\section{Agradecimentos}

O presente trabalho foi realizado com apoio da Coordenação de Aperfeiçoamento de Pessoal de Nível Superior - Brasil (CAPES) - Código de Financiamento 001 e do Conselho Nacional de Desenvolvimento Científico e Tecnológico (CNPq).

\section{Referências}

AVILA, C. et al. O Pensamento Computacional por meio da Robótica no Ensino Básico - Uma Revisão Sistemática. VI CONGRESSO BRASILEIRO DE INFORMÁTICA NA EDUCAÇÃO (CBIE 2017). Anais [...]. CBIE, 2017.

BACICH, Lilian; MORAN, José. Metodologias Ativas para uma Educação Inovadora: uma abordagem teórico-prática. Porto Alegre: Ed. Penso, 2018.

BARR, V., \& Stephenson, C. (2011). Bringing computational thinking to K-12: What is involved and what is the role of the computer science education community? ACM Inroads, 2(1), 48-54.

BOCCONI, S. et al. Developing Computational Thinking in Compulsory Educaction implications for policy and practice. Luxemburg: JRC Science for Policy Report; European Union, 2016.

EGUCHI, Amy. Robotics as a Learning Tool for Educational Transformation In: Proceedings of 4th International Workshop Teaching Robotics, Teaching with Robotics \& 5th International Conference Robotics in Education. Padova (Italy) July 18, 2014. ISBN 978-88-95872-06-3. pp. 27-34

IOANNOU, A. \& Makridou, E. Exploring the potentials of educational robotics in the development of computational thinking: A summary of current research and practical proposal for future work in: Education and Information Technologies (2018) 23: 2531. https://doi-org.ez45.periodicos.capes.gov.br/10.1007/s10639-018-9729-z

LIYING Xia, BAICHANG, Zhong. A systematic review on teaching and learning robotics content knowledge in K-12 in: Computers \& Education, Vol. 127, 2018, pag. 267-282, ISSN 0360-1315. https://doi.org/10.1016/j.compedu.2018.09.007. 
VIII Congresso Brasileiro de Informática na Educação (CBIE 2019)

Anais dos Workshops do VIII Congresso Brasileiro de Informática na Educação (WCBIE 2019)

LOPES, Daniel de Queiroz. A exploração de modelos e os níveis de abstração nas construções criativas com Robótica Educacional. Porto Alegre: UFRGS, 2008. 326f. Tese (Doutorado em Educação). Programa de Pós Graduação em Informática na Educação. Faculdade de Educação. Universidade Federal do Rio Grande do Sul, Porto Alegre, 2008.

mBlock - Programming Software Designed for STEAM Education. $<$ http://www.mblock.cc $>$

MORAN, José. Mudando a Educação com Metodologias Ativas. Coleção Mídias Contemporâneas. Convergências Midiáticas, Educação e Cidadania: aproximações jovens. Vol. II] Carlos Alberto de Souza e Ofelia Elisa Torres Morales (orgs.). PG: Foca Foto-PROEX/UEPG, 2015.

OLIVEIRA, José Antônio Colvara. Robótica como interface da tomada de consciência da ação e do conhecimento do objeto, através da metacognição como propulsora da produção do conhecimento. Porto Alegre: UFRGS, 2007. 114f. Tese (Doutorado em Educação). PPGIE. Universidade Federal do Rio Grande do Sul, Porto Alegre, 2007.

OLIVEIRA, Emiliano José Silva de; ARAÚJO, Ana Liz Souto Oliveira. Pensamento Computacional e Robótica: Um Estudo Sobre Habilidades Desenvolvidas em Oficina de Robótica Educacional. V CONGRESSO BRASILEIRO DE INFORMÁTICA NA EDUCAÇÃO (CBIE 2016). Anais [...]. CBIE, 2016.

PAPERT, Seymour. A Máquina das Crianças. Porto Alegre: Artmed, 2008. 220p.

PAPERT, Seymour. LOGO: computadores e educação. SP: Brasiliense, 1985. 254p.

PIAGET, Jean (e colaboradores). Abstração reflexionante: relações lógico-aritméticas e ordem das relações espaciais. Porto Alegre: Artes Médicas, 1995. 292p.

PIAGET, Jean. A Tomada de Consciência. São Paulo: Melhoramentos, 1977.

PIAGET, Jean. O nascimento da Inteligência na criança. RJ: Zahar, 1975. 389p.

POZO, J. I. A solução de problemas - aprender a resolver, resolver a para aprender. Porto Alegre: Artes Médicas, 1998.

RIBEIRO, Célia Rosa. Um estudo qualitativo sobre a robótica educativa no $1^{\mathrm{o}}$ ciclo do ensino Básico. 2006. 207p. Dissertação - Instituto de Educação e Psicologia / Universidade do Minho / PORTUGAL.

Robot Kits for Kids : mBot | Makeblock - Global STEAM Education Solution Provider. $<$ https://www.makeblock.com/steam-kits/mbot>

STEFFEN, Heloisa H. Robótica Pedagógica na Educação: um recurso de comunicação, regulagem e cognição. 2002. Dissertação - Escola de Comunicação e Artes da Universidade de São Paulo (ECA/USP). Orientador: Prof. Dr. Osvaldo Sangiorgi

WING, J. (2006). Computational thinking. Communications of the ACM, 49(3), 33-36.

YIN, Robert. Pesquisa qualitativa do início ao fim. Porto Alegre: Ed. Penso, 2016.

ZILLI, Silvana do Rocio. A Robótica Educacional no ensino fundamental: perspectivas e práticas. 2004. 89p. Dissertação - Programa de Pós Graduação em Engenharia da Produção / Universidade Federal de Santa Catarina. Orientadora: Ana Maria Franzoi. 